\title{
FIR-Based Phase Matching for Robust Retrospective-Cost Adaptive Control
}

\author{
E. Dogan Sumer, Matt H. Holzel, Anthony M. D’Amato, and Dennis S. Bernstein
}

\begin{abstract}
In this paper we develop frequency-domain methods for approximating IIR plants with FIR transfer functions. The underlying goal is to increase the performance and robustness of Retrospective-Cost Adaptive Control (RCAC), which is applicable to MIMO possibly nonminimum-phase (NMP) plants without the need to know the locations of the NMP zeros. The only required modeling information is an FIR approximation of the plant, which may be based on a limited number of Markov parameters, or possibly noisy frequency response data. In this paper we investigate the resulting phase mismatch between the true plant and the FIR approximation obtained through linear and nonlinear approximation methods. We consider degradation in the phase mismatch due to uncertainty in the frequency response data.
\end{abstract}

\section{INTRODUCTION}

Although there is no precise definition of adaptive control, it is generally understood to be a form of highly robust nonlinear control that does not a priori sacrifice performance for uncertainty. Adaptive controllers have been developed in continuous time and discrete time. Although most plants are naturally modeled in continuous time, the modeling data used by an adaptive controller is typically based on sampled data. In addition, most control applications are confined to a fixed, bounded sample rate, while controllers developed in discrete time can be directly transformed into embedded code. For these reasons, we consider direct digital adaptive control for sampled-data systems.

The direct digital adaptive control approach that we consider is retrospective-cost adaptive control (RCAC). This approach was developed in [1-5], where it was shown that RCAC requires a limited number of Markov parameters and knowledge of the nonminimum-phase (NMP) zeros, if any. This information is used to construct a finite-impulseresponse (FIR) approximation of the plant. With this limited modeling information, RCAC is applicable to stabilization, command following, and disturbance rejection for SISO and MIMO plants with arbitrary poles and zeros. In addition, RCAC requires limited plant modeling information and does not require knowledge of the poles, positive real or almost positive real assumptions, constraints on the allowable relative degree, persistent excitation, knowledge of the spectrum of the exogenous signal, or matching conditions on either the plant uncertainty or the exogenous disturbances.

The present paper focuses on the extension of RCAC given in [6-8], which removes the need to know the locations of the plant's NMP zeros. As shown in [6], the price paid for this relaxed modeling requirement is the need to ensure that

The authors are with the Department of Aerospace Engineering, The University of Michigan, Ann Arbor, MI 48109-2140. the Markov parameters used in RCAC provide a suitable approximation of the plant's frequency response. This insight suggests that an improved FIR approximation may be possible by directly approximating the frequency response rather than using Markov parameters. This possibility was explored in [8], where it was shown that, for a fixed FIR order, the Markov parameters may not provide the optimal FIR approximation of the plant's frequency response.

FIR approximation of IIR plants is studied in $[9,10]$, where an accurate state space realization is assumed to be available. In the present paper we focus on a related problem for SISO IIR plants where the available modeling information is possibly noisy frequency response data.

The goal of the present paper is to extend the results of [8] by constructing FIR approximations of the plant's frequency response subject to the given uncertainty. With these approximations, we can assess the transient and asymptotic performance of RCAC as a function of the accuracy of the approximation. This assessment allows us to relate the accuracy of the FIR approximation to the performance of RCAC, which in turn determines the modeling and identification accuracy needed to implement RCAC.

The contents of the paper are as follows. In Section 2, we present the adaptive control problem, and then summarize the RCAC algorithm. In Section 3, we present motivation for FIR approximation of IIR plants by illustrating the role of phase mismatch in performance properties of RCAC. In Sections 4 and 5, we develop linear and nonlinear parameterizations in order to fit the plant with an FIR model. Finally, in Section 6, we present numerical examples illustrating the use of the fit methods developed in Sections 4 and 5. The effect of noise in fit accuracy is also discussed.

\section{RCAC AdAPtive Control Problem}

\section{A. Problem Formulation}

Consider the MIMO discrete-time system

$$
\begin{aligned}
x(k+1) & =A x(k)+B u(k)+D_{1} w(k), \\
y(k) & =C x(k)+D_{2} w(k), \\
z(k) & =E_{1} x(k)+E_{0} w(k),
\end{aligned}
$$

where $k \geq 0, x(k) \in \mathbb{R}^{n}, z(k) \in \mathbb{R}^{l_{z}}$ is the measured performance, $y(k) \in \mathbb{R}^{l_{y}}$ contains additional measurements that are available for control, $u(k) \in \mathbb{R}^{l_{u}}$ is the input signal, $w(k) \in \mathbb{R}^{l_{w}}$ is the exogenous signal. 
The plant (1), (3) is represented by the transfer matrices

$$
\begin{aligned}
G_{z u}(z) & \triangleq E_{1}(z I-A)^{-1} B, \\
G_{z w}(z) & \triangleq E_{1}(z I-A)^{-1} D_{1}+E_{0} .
\end{aligned}
$$

Furthermore, for a positive integer $i$,

$$
H_{i} \triangleq E_{1} A^{i-1} B
$$

is the $i^{\text {th }}$ Markov parameter of $G_{z u}$.

The goal is to develop an adaptive output feedback controller that minimizes the performance variable $z$ in the presence of the exogenous signal $w$ with limited modeling information about (1)-(3). The components of the signal $w$ can represent either command signals to be followed, external disturbances to be rejected, or both, depending on the configurations of $D_{1}$ and $E_{0}$.

\section{B. Control Law}

We use the strictly proper time-series control law

$$
u(k)=\theta^{\mathrm{T}}(k) \phi(k-1),
$$

where

$$
\begin{aligned}
& \theta(k)=\left[\begin{array}{llllll}
N_{1}^{\mathrm{T}}(k) & \cdots & N_{n_{\mathrm{c}}}^{\mathrm{T}}(k) & M_{1}^{\mathrm{T}}(k) & \cdots & M_{n_{\mathrm{c}}}^{\mathrm{T}}(k)
\end{array}\right]^{\mathrm{T}},
\end{aligned}
$$

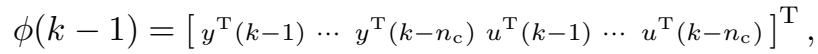

and, for all $1 \leq i \leq n_{\mathrm{c}}, N_{i}(k) \in \mathbb{R}^{l_{y} \times l_{u}}, M_{i}(k) \in \mathbb{R}^{l_{u} \times l_{u}}$. The control law (6) can be reformulated as

$$
u(k)=\Phi(k-1) \Theta(k),
$$

where

$$
\begin{aligned}
\Phi(k-1) & \triangleq I_{l_{u}} \otimes \phi^{\mathrm{T}}(k-1) \in \mathbb{R}^{l_{u} \times l_{u} n_{\mathrm{c}}\left(l_{u}+l_{y}\right)}, \\
\Theta(k) & \triangleq \operatorname{vec}(\theta(k)) \in \mathbb{R}^{l_{u} n_{\mathrm{c}}\left(l_{u}+l_{y}\right)},
\end{aligned}
$$

" $\otimes$ " denotes the Kronecker product, and "vec" is the columnstacking operator.

\section{Retrospective Performance}

For a positive integer $n_{\mathrm{f}}$, we define

$$
G_{\mathrm{f}}(\mathbf{q}) \triangleq D_{\mathrm{f}}^{-1}(\mathbf{q}) N_{\mathrm{f}}(\mathbf{q}),
$$

where

$$
\begin{array}{r}
N_{\mathrm{f}}(\mathbf{q}) \triangleq K_{1} \mathbf{q}^{n_{\mathrm{f}}-1}+K_{2} \mathbf{q}^{n_{\mathrm{f}}-2}+\cdots+K_{n_{\mathrm{f}}}, \\
D_{\mathrm{f}}(\mathbf{q}) \triangleq I_{l_{z}} \mathbf{q}^{n_{\mathrm{f}}}+A_{1} \mathbf{q}^{n_{\mathrm{f}}-1}+A_{2} \mathbf{q}^{n_{\mathrm{f}}-2}+\cdots+A_{n_{\mathrm{f}}},
\end{array}
$$

$K_{i} \in \mathbb{R}^{l_{z} \times l_{u}}$ for $1 \leq i \leq r, A_{j} \in \mathbb{R}^{l_{z} \times l_{z}}$ for $1 \leq j \leq r$, $n_{\mathrm{f}} \geq 1$ is the order of $G_{\mathrm{f}}$, and each polynomial entry of $D_{\mathrm{f}}(\mathbf{q})$ is asymptotically stable. Next, for $k \geq 1$, we define the retrospective performance variable

$$
\hat{z}(\hat{\Theta}(k), k) \triangleq z(k)+\Phi_{\mathrm{f}}(k-1) \hat{\Theta}(k)-u_{\mathrm{f}}(k),
$$

with

$$
\begin{aligned}
\Phi_{\mathrm{f}}(k-1) & \triangleq G_{\mathrm{f}}(\mathbf{q}) \Phi(k-1) \in \mathbb{R}^{l_{z} \times l_{u} n_{\mathrm{c}}\left(l_{u}+l_{y}\right)}, \\
u_{\mathrm{f}}(k) & \triangleq G_{\mathrm{f}}(\mathbf{q}) u(k) \in \mathbb{R}^{l_{z}},
\end{aligned}
$$

where $\hat{\Theta}(k)$ is determined by optimization below.

In this paper, $G_{\mathrm{f}}$ is chosen to be a finite-impulse-response (FIR) filter, that is, $A_{j}=0$, for $1 \leq j \leq n_{\mathrm{f}}$, as discussed in Section II-E. The role of $G_{\mathrm{f}}$ is discussed in Section III.

\section{Cumulative Cost and RCAC Update Law}

For $k>0$, we define the cumulative cost function

$$
\begin{aligned}
J(\hat{\Theta}(k), k) & \triangleq \sum_{i=1}^{k} \lambda^{k-i} \hat{z}^{\mathrm{T}}(\hat{\Theta}(k), i) \hat{z}(\hat{\Theta}(k), i) \\
& +\sum_{i=1}^{k} \lambda^{k-i} \eta(i) \hat{\Theta}^{\mathrm{T}}(k) \Phi_{\mathrm{f}}^{\mathrm{T}}(i-1) \Phi_{\mathrm{f}}(i-1) \hat{\Theta}(k) \\
& +\lambda^{k}\left(\hat{\Theta}(k)-\Theta_{0}\right)^{\mathrm{T}} P_{0}^{-1}\left(\hat{\Theta}(k)-\Theta_{0}\right),
\end{aligned}
$$

where $\lambda \in(0,1], P_{0} \in \mathbb{R}^{l_{u} n_{\mathrm{c}}\left(l_{u}+l_{y}\right) \times l_{u} n_{\mathrm{c}}\left(l_{u}+l_{y}\right)}$ is positive definite, $\eta(k) \triangleq \eta_{0} z^{\mathrm{T}}(k) z(k) \geq 0$, and $\Theta_{0} \in \mathbb{R}^{l_{u} n_{\mathrm{c}}\left(l_{u}+l_{y}\right)}$.

The following result follows from the RLS theory [11].

Proposition 2.1: Let $P(0)=P_{0}$ and $\Theta(0)=\Theta_{0}$. Then, for all $k \geq 1$, the cumulative cost function (17) has a unique global minimizer $\Theta(k)$. Furthermore, $\Theta(k)$ is given by

$$
\begin{aligned}
\Theta(k)= & {\left[I-K(k) \Phi_{\mathrm{f}}(k-1)\right] \Theta(k-1) } \\
& -P(k) \Phi_{\mathrm{f}}^{\mathrm{T}}(k-1)\left[z(k)-u_{\mathrm{f}}(k)\right],
\end{aligned}
$$

where $P(k)$ satisfies

$$
P(k)=\frac{1}{\lambda}\left[P(k-1)-K(k) \Phi_{\mathrm{f}}(k-1) P(k-1)\right],
$$

and

$$
\begin{aligned}
K(k) \triangleq & P(k-1) \Phi_{\mathrm{f}}^{\mathrm{T}}(k-1) \\
& \cdot\left[\frac{\lambda}{1+\eta(k)} I_{l_{z}}+\Phi_{\mathrm{f}}(k-1) P(k-1) \Phi_{\mathrm{f}}^{\mathrm{T}}(k-1)\right]^{-1} .
\end{aligned}
$$

\section{E. Phase-Matching-Based Construction of $G_{\mathrm{f}}$}

For $\Omega \in[0, \pi] \mathrm{rad} / \mathrm{sample}$, the phase mismatch $\Delta(\Omega)$ between $G_{\mathrm{f}}$ and $G_{z u}$ is defined by

$$
\Delta(\Omega) \triangleq \cos ^{-1} \frac{\operatorname{Re}\left[G_{z u}\left(e^{\jmath \Omega}\right) \overline{G_{\mathrm{f}}\left(e^{\jmath \Omega}\right)}\right]}{\left|G_{z u}\left(e^{\jmath \Omega}\right)\right|\left|G_{\mathrm{f}}\left(e^{\jmath \Omega}\right)\right|} \in[0,180] .
$$

Note that $\Delta(\Omega)$ represents the angle between $G_{z u}\left(e^{\jmath \Omega}\right)$ and $G_{\mathrm{f}}\left(e^{\jmath \Omega}\right)$ in the complex plane. For the phase-matching-based construction, $G_{\mathrm{f}}$ is chosen to satisfy

$$
\Delta(\Omega) \leq 90 \mathrm{deg} \text {, for all } \Omega \in[0, \pi] \mathrm{rad} / \text { sample. }
$$

A weaker condition is sufficient when $G_{z u}$ is asymptotically stable, and the exogenous signal $w(k)$ is harmonic. In this case, the phase-matching-based construction requires

$$
\Delta(\Omega) \leq 90 \operatorname{deg}, \Omega \in \operatorname{spec}(w),
$$


where $" \operatorname{spec}(w) "$ is the frequency spectrum of $w$. Optimization methods for minimizing the phase mismatch are presented in Sections IV, V for SISO plants.

\section{Motivating EXAMPLE}

In this section, we present an example that illustrates the role of phase matching $\Delta(\theta)$ on the performance of RCAC. In particular, numerical examples in [8] suggest that $\Delta(\theta) \leq$ $90 \mathrm{deg}$ in the exogenous signal frequencies is a sufficient condition for convergence of $z$ to zero.

Example 3.1: Consider the NMP plant $G_{z u}$ with $d=1$, $H_{1}=1$, poles $0.9,0.5 \pm \jmath 0.5$, and NMP zeros 1.3, 1.4. We consider the sinusoidal command $w(k)=2 \sin \theta_{1} k$, where $\theta_{1}=0.285 \mathrm{rad} /$ sample. We take $G_{\mathrm{f}}(\mathbf{q})=H_{1} \mathbf{q}^{-1}+$ $\mathrm{H}_{2} \mathbf{q}^{-2}+\mathrm{H}_{3} \mathbf{q}^{-3}$, and thus the phase mismatch at the command frequency is $\Delta\left(\theta_{1}\right)=62 \mathrm{deg}$. We let $n_{\mathrm{c}}=5$, $P_{0}=0.1 I$, and $\eta_{0}=1$. The performance $z(k)$ converges to zero as shown in Figure 1(a).

Keeping $G_{\mathrm{f}}$ the same, we now consider the step command $w(k)=2 \operatorname{step}(k)$. The phase mismatch at DC is $\Delta(0)=180$ deg. We let $n_{\mathrm{c}}=5, P_{0}=0.1 I$, and $\eta_{0}=1$. The performance $z(k)$ is driven in the opposite direction because of the 180deg phase mismatch, and thus does not converge to zero, as shown in Figure 1(b).

We now choose $G_{\mathrm{f}}(\mathbf{q})=0.34 \mathbf{q}^{-1}-0.11 \mathbf{q}^{-2}+0.85 \mathbf{q}^{-3}$, and consider the step command $w(k)=2 \operatorname{step}(k)$. Note that $G_{\mathrm{f}}$ is not constructed using the Markov parameters. The phase mismatch at DC is now $\Delta(0)=0$ deg. We let $n_{\mathrm{c}}=5$, $P_{0}=0.1 I$, and $\eta_{0}=1$. The performance now converges to zero as shown in Figure 1(c).

Next, we keep $G_{\mathrm{f}}$ the same, but consider the sinusoidal command $w(k)=2 \sin \theta_{1} k$, where $\theta_{1}=0.285 \mathrm{rad} / \mathrm{sample}$. We now have $\Delta\left(\theta_{1}\right)=144 \mathrm{deg}$. We let $n_{\mathrm{c}}=5, P_{0}=0.1 I$, and $\eta_{0}=1$. The performance $z(k)$ does not converge to zero as shown in Figure 1(d).

\section{Linear FIR Fitting Method}

In this section, we formulate a constrained linear least squares method that fits the frequency response of $G_{z u}$ with an FIR transfer function $G_{\mathrm{f}}$. We assume that an estimate of the frequency response of the plant $G_{z u}\left(e^{\jmath \theta}\right)$ is available at a finite number of frequencies. This knowledge can be obtained through either modeling or frequency-domain identification. We constrain the least squares solution to bound the phase mismatch $\Delta(\theta)$ over a chosen frequency interval $\left[\theta_{l}, \theta_{h}\right]$, where $0 \leq \theta_{l}<\theta_{h} \leq \pi$. The phase mismatch bound does not need to be uniform over $\left[\theta_{l}, \theta_{h}\right]$. Furthermore, the largest bound that we allow is $90 \mathrm{deg}$. This is consistent with the numerical results of [8], which suggests that a sufficient condition for RCAC to drive the performance to zero is to have $\Delta(\theta) \leq 90 \mathrm{deg}$ for all $\theta \in[0, \pi]$. Finally, we show that the numerator coefficients of $G_{\mathrm{f}}$ obtained by using the unconstrained least squares solution are Markov parameters of $G_{z u}$. However, this is not the case when phase constraints are imposed.
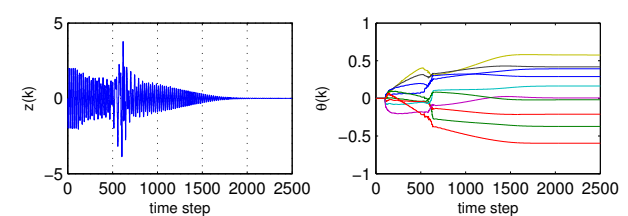

(a)
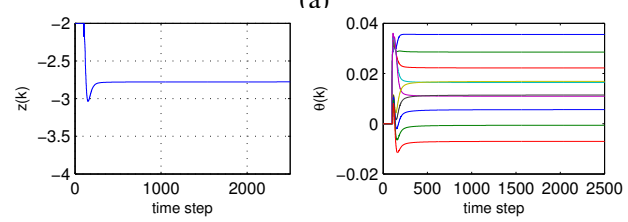

(b)
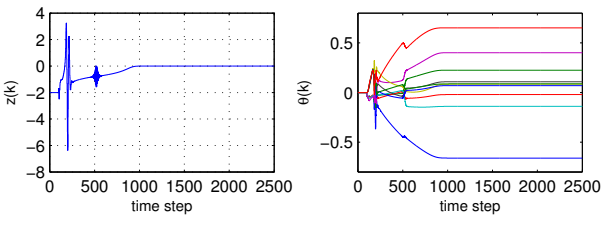

(c)

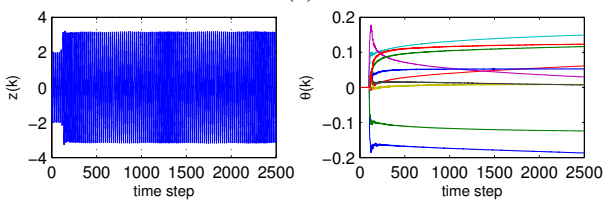

(d)

Fig. 1. Example 3.1. (a) shows the closed-loop response to the sinusoidal command $w(k)=2 \sin 0.285 k$ with $\Delta(0.285)=62 \mathrm{deg}$, (b) shows the closed-loop response to the step command $w(k)=2$ with $\Delta(0)=180$ deg, (c) shows the closed-loop response to the step command $w(k)=2(k)$ with $\Delta(0)=0 \mathrm{deg}$, and finally, (d) shows the closed-loop response to the step command $w(k)=2 \sin (0.285 k)$ with $\Delta(0)=144 \mathrm{deg}$. The performance converges to zero for the cases where $\Delta(\theta)<90 \mathrm{deg}$.

\section{A. Linear Least Squares Parametric Model}

For $1 \leq i \leq N$, consider the frequency response estimates

$$
\hat{G}_{z u}\left(e^{\jmath \theta_{i}}\right)=\alpha_{i}+\jmath \beta_{i},
$$

at the frequencies $\theta_{i}$, where $\alpha_{i} \in \mathbb{R}, \beta_{i} \in \mathbb{R}$. We want to fit the above estimates with the FIR transfer function

$$
G_{\mathrm{f}}(z) \triangleq \frac{\kappa_{1} z^{s-1}+\cdots+\kappa_{s-1} z+\kappa_{s}}{z^{s}},
$$

where $s$ is the order of the FIR model, and $\kappa_{i} \in \mathbb{R}$ are the corresponding numerator coefficients that will be determined. We now expand (25) into the Laurent series

$$
G_{\mathrm{f}}(z)=\kappa_{1} z^{-1}+\cdots+\kappa_{s} z^{s}
$$

which is finite since $G_{\mathrm{f}}$ is an FIR model. We now evaluate (26) at $e^{\theta_{i}}$ for $i=1, \ldots, N$, and separate the unknown parameters $\kappa_{i}$ to obtain the linear parametric model

$$
\begin{aligned}
\left.G_{\mathrm{f}}(z)\right|_{e^{\jmath \theta_{i}}} & =\kappa_{1} e^{-\jmath \theta_{i}}+\cdots+\kappa_{s} e^{-\jmath s \theta_{i}} \\
& =\left[\begin{array}{lll}
\kappa_{1} & \cdots & \kappa_{s}
\end{array}\right]\left(\begin{array}{lll}
\cos \theta_{i} & \cdots & \cos \left(s \theta_{i}\right)
\end{array}\right]^{\mathrm{T}} \\
& \left.+\jmath\left[\begin{array}{lll}
-\sin \theta_{i} & \cdots & -\sin \left(s \theta_{i}\right)
\end{array}\right]^{\mathrm{T}}\right) .
\end{aligned}
$$

Then, the linear least squares fit of (24) with the FIR parametric model (27) is obtained by minimizing

$$
\min _{X}\left\|Y-\Phi^{\mathrm{T}} X\right\|
$$


where

$$
\begin{aligned}
& Y=\left[\begin{array}{llllll}
\alpha_{1} & \cdots & \alpha_{N} & \beta_{1} & \cdots & \beta_{N}
\end{array}\right]^{\mathrm{T}}, \\
& \Phi=\left[\begin{array}{cccccc}
\cos \theta_{1} & \cdots & \cos \theta_{N} & -\sin \theta_{1} & \cdots & -\sin \theta_{N} \\
\vdots & & \vdots & \vdots & & \vdots \\
\cos \left(s \theta_{1}\right) & \cdots & \cos \left(s \theta_{N}\right) & -\sin \left(s \theta_{1}\right) & \cdots & -\sin \left(s \theta_{N}\right)
\end{array}\right] \text {, } \\
& X=\left[\begin{array}{lll}
\kappa_{1} & \cdots & \kappa_{s}
\end{array}\right]^{\mathrm{T}} .
\end{aligned}
$$

\section{B. Phase Mismatch Constraints}

To impose phase mismatch bounds on the solution $X$ of (28), we consider the constrained linear least squares problem

$$
\min _{X}\left\|Y-\Phi^{\mathrm{T}} X\right\|, \quad \text { subject to } \quad \mathcal{C} X \leq 0,
$$

where $\mathcal{C}$ is constructed based on the phase information of each $\hat{G}_{z u}\left(e^{\jmath \theta_{i}}\right)$ and the desired phase mismatch bounds at each frequency.

For example, consider the frequency-response estimate $\hat{G}_{z u}\left(e^{\jmath \theta_{m}}\right)=\alpha_{m}+\jmath \beta_{m}$, and the phase mismatch bound $\bar{\Delta}_{m} \in(0,90)$ deg imposed on $\Delta(\theta)$ at the frequency $\theta_{m}$. Then, the linear phase mismatch bounds $\operatorname{Im}(x)=u_{m} \operatorname{Re}(x)$ and $\operatorname{Im}(x)=l_{m} \operatorname{Re}(x)$ are determined by $\bar{\Delta}_{m}$ as shown in Figure 2. The slopes $u_{m}$ and $l_{m}$ are given by

$$
\begin{gathered}
u_{m} \triangleq \tan \left(\angle\left(\alpha_{m}+\jmath \beta_{m}\right)+\bar{\Delta}_{m}\right), \\
l_{m} \triangleq \tan \left(\angle\left(\alpha_{m}+\jmath \beta_{m}\right)-\bar{\Delta}_{m}\right) .
\end{gathered}
$$

Since $u_{m} \geq \frac{\beta_{m}}{\alpha_{m}} \geq l_{m}$, and $\alpha_{m}>0$, we have the inequality constraints $\beta_{m}-u_{m} \alpha_{m} \leq 0$ and $l_{m} \alpha_{m}-\beta_{m} \leq 0$. Then, the linear constraint that needs to be imposed on $X$ to bound $\Delta\left(\theta_{m}\right)$ by $\bar{\Delta}_{m}$ is given by

$$
\mathcal{C}_{m} X \leq 0, \quad \mathcal{C}_{m}=\left[\begin{array}{c}
l_{m} \Phi^{\mathrm{T}}(m,:)-\Phi^{\mathrm{T}}(N+m,:) \\
\Phi^{\mathrm{T}}(N+m,:)-u_{m} \Phi^{\mathrm{T}}(m,:)
\end{array}\right],
$$

and $\Phi^{\mathrm{T}}(m,:)$ represents the $m^{\text {th }}$ row of $\Phi^{\mathrm{T}}$.

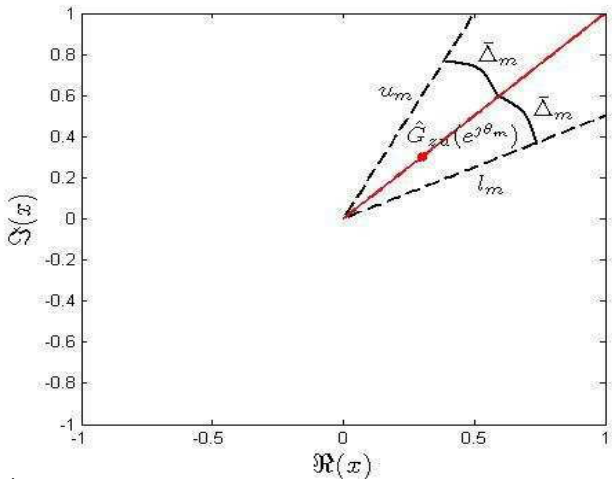

Fig. 2. $\hat{G}_{z u}\left(e^{\jmath \theta_{m}}\right)$, the linear upper bound $u_{m}$ and the linear lower bound $l_{m}$. Note that $u_{m}$ and $l_{m}$ are the slopes of the dashed black lines, and are given by (33), (34) respectively.

The above procedure is carried out for each frequency $\theta_{1}, \ldots, \theta_{N}$ to construct the constraint matrix

$$
\mathcal{C}=\left[\begin{array}{lll}
\mathcal{C}_{1} & \cdots & \mathcal{C}_{N}
\end{array}\right]^{\mathrm{T}} \in \mathbb{R}^{2 N \times s} .
$$

Note that there are 4 possible constraint inequality conditions corresponding to 12 possible configurations of $u_{m}$ and $l_{m}$, all of which are illustrated in Figure 3.
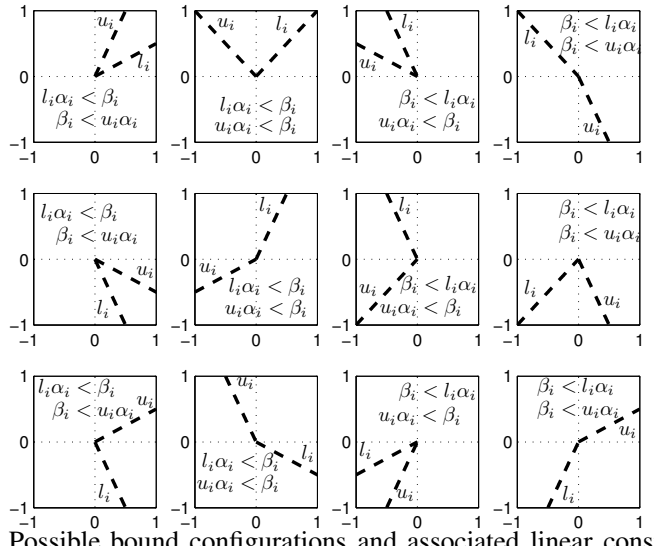

Fig. 3. Possible bound configurations ${ }^{-1}$ and associated linear constraints.

\section{Special Case: Unconstrained Least Squares Solution}

We now illustrate the unconstrained least squares solution of (28) with exact frequency response estimates (24) of $G_{z u}$. Consider $G_{z u}(z)=$ $12 \frac{(z-1.4)(z-0.6)(z-0.4)}{(z-0.85)(z-0.5)(z-0.3+0.8 \jmath)(z-0.3-0.8))}$. We apply unconstrained least squares fitting using exact frequency response estimates $\hat{G}_{z u}\left(e^{\jmath \theta_{i}}\right)=G_{z u}\left(e^{j \theta_{i}}\right)$, where $\theta_{i}$ are equally placed between 0 and $\pi$. We let the order of $G_{\mathrm{f}}$ be $s=10$. Figure 4 shows that the estimated coefficients $\kappa_{1}, \ldots, \kappa_{10}$ converge to the Markov parameters $H_{1}, \ldots, H_{10}$ as the number of data points used in the estimation is increased.

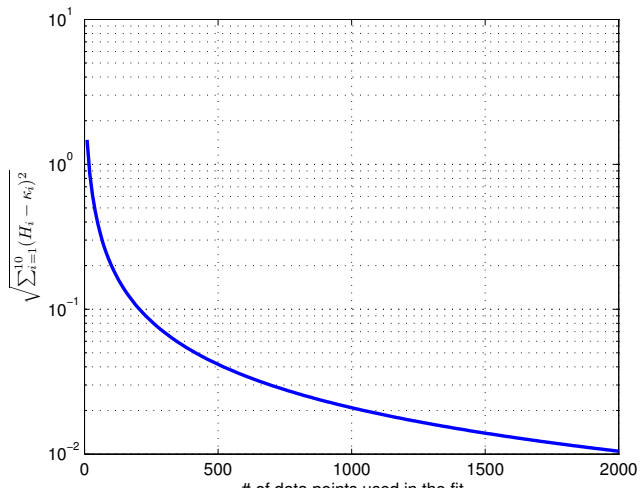

Fig. 4. Unconstrained linear \# fl data points used in the fit squares solution converges to Markov parameters of $G_{z u}$.

\section{Nonlinear FIR FitTing Method}

We now develop a nonlinear parameterization to fit the phase plot of $G_{z u}$ with the phase of an FIR transfer function. This nonlinear formulation requires only an estimate of the phase plot of $G_{z u}$ in $\left[\theta_{l}, \theta_{h}\right]$, and thus it requires less modeling information than the linear method presented in the previous section.

For $1 \leq i \leq N$, let

$$
\phi\left(\theta_{i}\right) \triangleq \angle \hat{G}_{z u}\left(e^{\jmath \theta_{i}}\right) \in[0,360),
$$

be the estimates of the phase of $G_{z u}$ at frequencies $0 \leq \theta_{1} \leq$ $\ldots \leq \theta_{N} \leq \pi$. We want to fit the above phase estimates using the phase plot of the FIR model (25). Let $g\left(\theta_{i}\right)$ denote the phase $\angle G_{\mathrm{FIR}}\left(e^{\jmath \theta}\right)$ of the FIR model evaluated at the 
frequency $\theta_{i}$. Then, it follows from (27) that

$$
\begin{aligned}
g\left(\theta_{i}\right) & =\angle\left(\kappa_{1} e^{-\jmath \theta_{i}}+\cdots+\kappa_{s} e^{\jmath s \theta_{i}}\right) \\
& =\angle \sigma_{i}+\jmath \omega_{i} \\
& =\operatorname{atan} 2\left(\omega_{i}, \sigma_{i}\right)
\end{aligned}
$$

where

$$
\sigma_{i}=\sum_{l=1}^{s} \kappa_{l} \cos \left(l \theta_{i}\right), \quad \omega_{i}=-\sum_{l=1}^{s} \kappa_{l} \sin \left(l \theta_{i}\right),
$$

and atan 2 is the four-quadrant inverse of the tangent function mapped to $[0,360)$. Then, the phase mismatch $\Delta\left(\theta_{i}\right)$ is

$$
\begin{aligned}
& \Delta\left(\theta_{i}\right)=\min \left(\phi\left(\theta_{i}\right)-g\left(\theta_{i}\right) \quad \bmod 360,\right. \\
& \left.360-\left(\phi\left(\theta_{i}\right)-g\left(\theta_{i}\right) \quad \bmod 360\right)\right) .
\end{aligned}
$$

Now, solving the minimization problem

$$
\min _{\kappa_{i}} \sum_{l=1}^{N}\left\|\Delta\left(\theta_{l}\right)\right\|
$$

yields the numerator coefficients $\kappa_{i}$ of the FIR approximation $G_{\mathrm{FIR}}$ that provides the best phase matching with the estimated phase plot (35) of $G_{z u}$.

\section{FIR FITTING EXAMPLES}

We now present numerical examples illustrating the use of linear and nonlinear fitting methods for minimizing $\Delta(\theta)$ We use numerical optimization tools for both linear anc nonlinear fitting; we use the Matlab functions lsqlin for minimizing (32), and lsqnonlin for minimizing (37).

The constrained minimization problem (32) may not have a solution if the chosen order of the FIR fit is too smal to satisfy the constraints. If that is the case, we increase the order of the FIR fit until the minimization problem is feasible.

Since we are using only the phase information (35) in the nonlinear method, scaling each coefficient $\kappa_{i}$ by a positive constant $\gamma$ results in the same cost value (37). In order to avoid numerical problems that can arise, we first fix $\kappa_{1}=1$, and solve for $\kappa_{i}, 2 \leq i \leq s$. Next, we fix $\kappa_{1}=-1$, and repeat the process. Finally, we compare the residuals and take the solution with the smaller residual norm.

Example 6.1: Consider the plant $G_{z u}(z)$ with $H_{1}=1$, poles $0.1,0.5 \pm 0.3,0.85,0$, minimum-phase zero 0.45 , and NMP zeros 1.5, $1.2 \pm 0.5$. We first assume we have the exact knowledge of the frequency response $G_{z u}\left(e^{j \theta_{i}}\right)$ at 1001 equally spaced frequencies in $[0, \pi]$. With this knowledge, we apply linear fitting to uniformly bound the phase mismatch $\Delta(\theta)$ by $\bar{\Delta}_{1}=80 \mathrm{deg}, \bar{\Delta}_{2}=40 \mathrm{deg}$, and $\bar{\Delta}_{3}=10$ deg. Figure 5 shows the phase mismatch functions of the resulting FIR approximations. Note that the order of the FIR fit increases as the phase mismatch bound becomes tighter.

We now assume that we do not have complete frequency response information of $G_{z u}$, but we do have exact knowledge of the phase plot at the above specified frequencies. With this knowledge, we apply nonlinear fitting to minimize $\Delta(\theta)$ over $[0, \pi]$. Figure 6 shows the phase mismatch func- tions of the resulting FIR approximations with orders $s=4$, 5 , and 7 .

We now assume we have no frequency domain knowledge of $G_{z u}$, but we have exact knowledge of Markov parameters. With this knowledge, we construct $4^{\text {th }}, 5^{\text {th }}$ and $7^{\text {th }}$-order FIR plants, the phase mismatch functions of which are illustrated in Figure 7. Note that $\Delta(0)=180 \mathrm{deg}$ in each case.
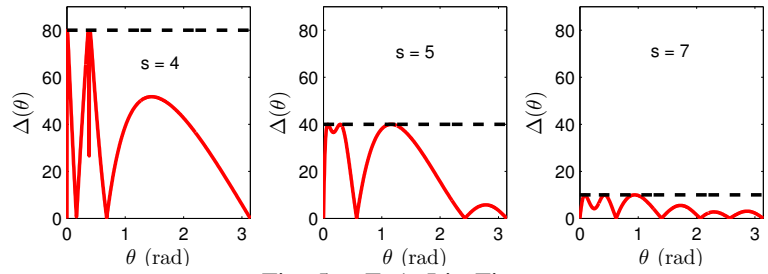

Fig. 5. Ex1: Lin Fit.
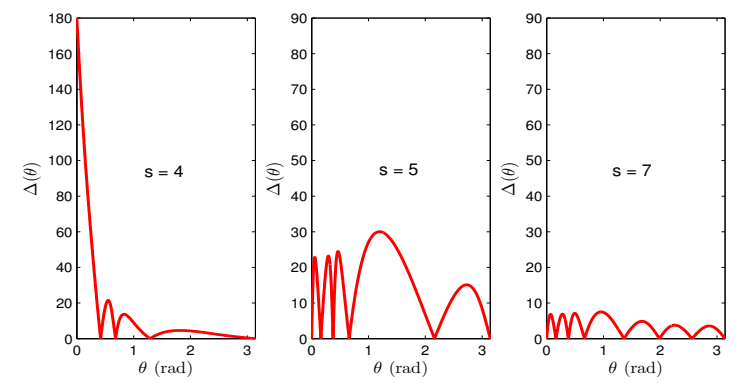

Fig. 6. Ex1: NonLin Fit.
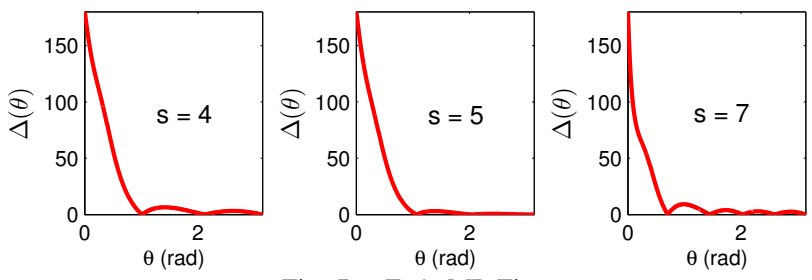

Fig. 7. Ex1: MP Fit.

Example 6.2: Consider the same plant $G_{z u}$ as in Example 6.1, and assume we have the exact knowledge of the frequency response $G_{z u}\left(e^{\theta_{i}}\right)$ at 1001 equally spaced frequencies in $[0, \pi]$. Suppose we are interested in tracking sinusoids at a particular frequency range with RCAC. Therefore, the objective is to impose nonuniform bounds that are tighter at the frequencies of interest. In particular, we consider three cases: smaller mismatch at low frequencies, smaller mismatch at high frequencies, and smaller mismatch at intermediate frequencies. Furthermore, to have robustness at all frequencies, we want $\Delta(\theta)<90$ deg for all $\theta \in[0, \pi]$. We apply linear fitting with nonuniform bounds to obtain the phase mismatch functions illustrated in Figure 8. The order of the FIR fit is $s=5$ in each case, which is smaller than the order $s=7$ we obtain when we impose a uniform 10 deg bound at every frequency.
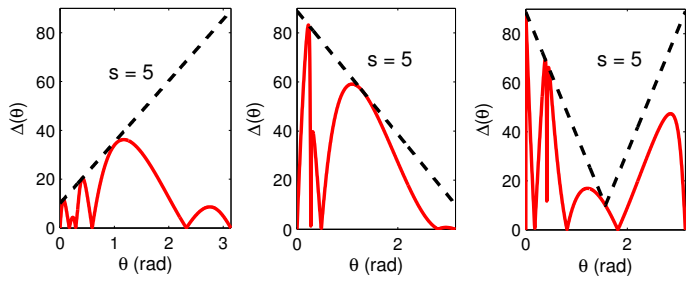

Fig. 8. Ex2: Lin Fit. 
Example 6.3: Consider the same plant $G_{z u}$ as above. We now assume that the plant model is unknown. With the plant realized in controllable canonical form, we excite the unknown plant with a white noise sequence and collect output measurements for 2500 time steps with the unknown nonzero initial condition $x(0)=$ $\left[\begin{array}{lllll}0.8644 & 0.0942 & -0.8519 & 0.8735 & -0.4380\end{array}\right]^{\mathrm{T}}$. We then take the ratio of the fast fourier transforms of the output and input signals to obtain frequency response estimates $\hat{G}_{z u}\left(e^{\jmath \theta}\right)$ over $\theta \in[0, \pi]$. The bode plot of $G_{z u}$ and the estimated frequency response are shown in Figure 9.
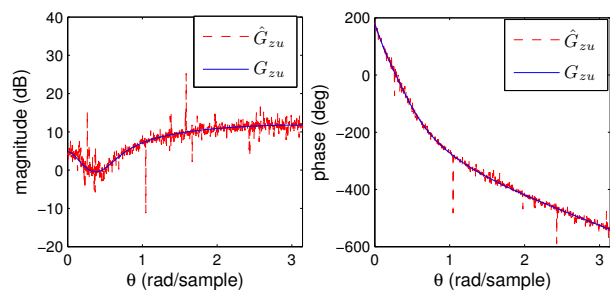

Fig. 9. Ex2: Lin Fit.

Using the noisy frequency response estimates, we first apply the linear fitting method with a uniform phase mismatch bound $\bar{\Delta}=80 \mathrm{deg}$. Figure 10 shows the phase plot of the resulting FIR transfer function $G_{\mathrm{f}}$ and the phase mismatch function $\Delta(\theta)$ between the actual plant $G_{z u}$ and $G_{\mathrm{f}}$. Although the phase of $G_{\mathrm{f}}$ is within a \pm 80 deg envelope of the estimated phase plot $\angle \hat{G}_{z u}$, the phase mismatch with $G_{z u}$ gets above 80 deg near $\theta=1 \mathrm{rad} /$ sample. Furthermore, the order of $G_{\mathrm{FIR}}$ is now $s=11$, which is larger than the order $s=4$ we obtain if the frequency response estimates are exact.
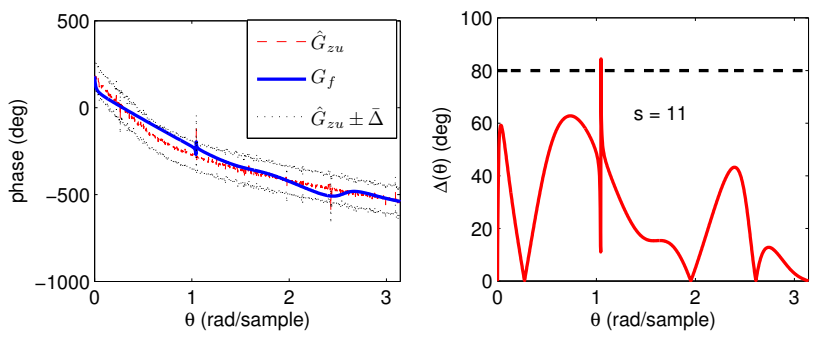

Fig. 10. Ex2: Lin Fit.

Using the estimated phase plot, we now apply the nonlinear fitting method to minimize $\bar{\Delta}$. Figure 11 shows the phase plot of the resulting FIR transfer function $G_{\mathrm{FIR}}$ and the phase mismatch function $\Delta(\theta)$ between the actual plant $G_{z u}$ and $G_{\mathrm{FIR}}$. Since the nonlinear method directly minimizes the phase difference between the estimated phase plot and the $G_{\text {FIR }}$ model in a least squares sense, the effect of noise is less significant than the linear method, and $G_{\mathrm{FIR}}$ matches the actual plant with less than 30-deg phase mismatch for all $\theta \in[0, \pi]$. Furthermore, the order of $G_{\mathrm{FIR}} s=8$ is lower than the order $s=11$ we obtain with the linear method.

\section{CONCLUSIONS}

We provided frequency-domain fitting methods for approximating IIR plants with FIR transfer functions. In particular, we developed a linear parameterization that fits the frequency response estimates of the IIR plant with an FIR transfer function, as well as a nonlinear parameterization
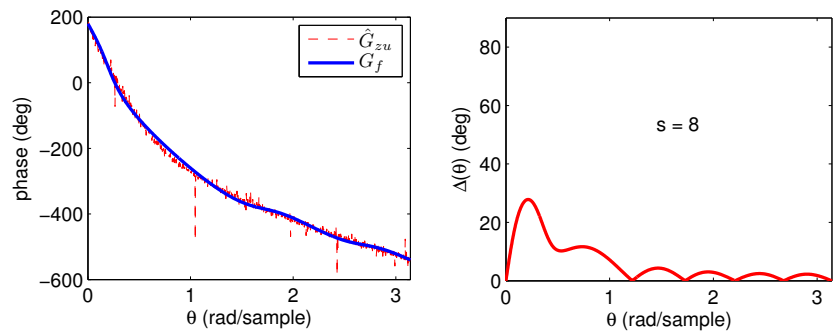

Fig. 11. Ex: NonLin Fit.

that fits the phase plot of the IIR plant with the phase of an FIR transfer function. We showed that in the absence of phase constraints, the solution of the linear fitting method converges to Markov parameters of the plant. We provided a motivating example illustrating the role of phase matching in on the performance of RCAC. We compared the linear and nonlinear methods in terms of phase matching performance, and investigated the degradation in phase matching when the frequency response estimates are noisy.

\section{REFERENCES}

[1] J. B. Hoagg and D. S. Bernstein, "Retrospective Cost Model Reference Adaptive Control for Nonminimum-Phase Discrete-Time Systems, Part 1: The Ideal Controller and Error System; Part 2: The Adaptive Controller and Stability Analysis," Proc. Amer. Contr. Conf., pp. 29272938, San Francisco, CA, June 2011.

[2] J. B. Hoagg and D. S. Bernstein, "Retrospective Cost Adaptive Control for Nonminimum-Phase Discrete-Time Systems Part 1: The Ideal Controller and Error System; Part 2: The Adaptive Controller and Stability Analysis," Proc. Conf. Dec. Contr., pp. 893-904, Atlanta, GA, December 2010.

[3] R. Venugopal and D. S. Bernstein, "Adaptive Disturbance Rejection Using ARMARKOV System Representations," IEEE Trans. Contr. Sys. Tech., vol. 8, pp. 257-269, 2000.

[4] M. A. Santillo and D. S. Bernstein, "Adaptive Control Based on Retrospective Cost Optimization", AIAA J. Guid. Contr. Dyn., vol. 33, pp. 289-304, 2010.

[5] J. B. Hoagg, M. A. Santillo and D. S. Bernstein, "Discrete-Time Adaptive Command Following and Disturbance Rejection for MinimumPhase Systems with Unknown Exogenous Dynamics," IEEE Trans. Autom. Contr., vol. 53, pp. 912-928, 2008.

[6] A. M. D'Amato, E. D. Sumer, and D. S. Bernstein, "FrequencyDomain Stability Analysis of Retrospective-Cost Adaptive Control for Systems with Unknown Nonminimum-Phase Zeros," Proc. Conf. Dec. Contr., Orlando, FL, December 2011.

[7] A. M. D'Amato, E. D. Sumer, K. S. Mitchell, A. V. Morozov, J. B. Hoagg and D. S. Bernstein, "Adaptive Output Feedback Control of the NASA GTM Model with Unknown Nonminimum-Phase Zeros," AIAA Guid. Nav. Contr. Conf., Portland, OR, August 2011, AIAA2011-6204

[8] E. D. Sumer, A. M. D'Amato and D. S. Bernstein, "Robustness of Retrospective-Cost Adaptive Control to Markov-Parameter Uncertainty," Proc. Conf. Dec. Contr., Orlando, FL, December 2011.

[9] L. Chai, J. Zhang, C. Zhang, and E. Mosca , "From IIR to FIR Digital MIMO Models: A Constructive Hankel Norm Approximation Method," Proc. Conf. Dec. Contr., pp. 5893-5898, Seville, Spain, December 2005.

[10] Y. Yamamato, B. D. O. Anderson, M. Nagahara, and Y. Koyanagi, "Optimizing FIR Approximation for Discrete-Time IIR Filters," IEEE Signal Processing Letters, vol. 10, no. 9, pp. 273-276, September 2003.

[11] K. J. Astrom and B. Wittenmark, Adaptive Control, 2nd ed., AddisonWesley, 1995. 